It's cloning again!

\section{It's cloning again!}

Nikola Biller-Andorno

\section{Further discussion of the ongoing human cloning debate.}

n the late 1990s cloning was still the subject of passionate debate. While philosophers were crossing swords about the implications of the "Dolly technique" for the meaning of human identity, sweeping declarations were made by major international bodies such as the World Medical Association, UNESCO, and the World Health Organization that unanimously condemned human reproductive cloning as ethically unacceptable and/or contrary to human dignity. By now, the topic elicits a mere frown, sneer, sigh, or yawn from most bioethicists, depending on their temperament or mood. The same may hold true for those delegates at the United Nations who sat through many sessions that included the ominous agenda item entitled "international convention against reproductive cloning of human beings".

Human cloning became an issue at the United Nations when the item was introduced at the request of France and Germany in 2001. The two countries limited their proposal to reproductive cloning. This move, although aiming quite reasonably at the formulation of a minimal consensus among member states, got at least Germany into some trouble at home, as critical voices from different parts of the political spectrum argued that Germany should rather pursue a comprehensive ban on human cloning for any purpose, which would be more in accordance with its own national laws. This criticism was largely an echo of the resistance that the French-German proposal encountered at the UN, where the United States, the Vatican, Costa Rica, and other countries championed a total ban on the use of human embryos, regardless of its purpose, including medical and scientific research.

A remarkable sort of culture war has been going on at the UN headquarters in New York ever since, observed by stakeholders, bioethicists and the public at large, with an interest that was rekindled whenever the occasional report of a cloned baby about to be born went through the press. The main arguments of the front lines ran as follows: All forms of human cloning violate human rights and the intrinsic dignity of human beings and need to be outlawed, according to the proponents of a comprehensive ban. This includes cloning for research purposes, which is morally unacceptable because it involves the creation and destruction of human embryos, thus instrumentalizing and eventually taking a human life. Also, if cloning for non-reproductive purposes goes on, reproductive cloning will inevitably follow, particularly since the methods leading to the creation of the human embryo and its cultivation up to the blastocyst stage are the same.

The proponents of a partial ban countered at the political, rather than philosophical level. The existing difference of opinion among member statesand, in fact, of national laws-needed to be taken into account. What use was a comprehensive, but controversial convention that an important number of states would not ratify? In order to prevent the cloning techniques from being used for reproductive purposes a convention of universal scope was needed-and that was feasible only if the convention were limited to reproductive cloning, where broad consensus existed among member states.

An Ad Hoc Committee, a series of working groups and much talk behind the scenes could not resolve the fundamental differences. In 2003 the drafting was delayed for another year due to lack of consensus, following a procedural motion introduced by the Organization of the Islamic Conference. In 2004, a vote was expected between a draft introduced by Costa Rica including a comprehensive ban and a proposal for a partial ban, which had been put forward by Belgium and would have banned reproductive cloning while allowing countries to choose between banning therapeutic cloning, putting a moratorium on the practice or regulating it through national legislation to prevent misuse. The continuation of the debate was delayed until after the U.S. election, and by midNovember the idea of a convention was abandoned in favor of a less binding declaration whose wording was "ambiguous enough to please everybody", as an insider commented. The chairman of the Sixth (Legal) Committee, where the cloning issue was mainly being dealt with, proposed a working group that would finalize the text of a declaration based on a draft resolution that Italy had submitted. The chairman's suggestion was approved by consensus on 19 November 2004, and the working group has been scheduled to meet on 14 and 15 February 2005, with the Sixth Committee considering the work of the group on 18 February 2005.

One does not have to be a cynic to come away with the impression that the effort and resources are somewhat out of proportion compared to the output; particularly if the declaration will indeed include the formulation suggested in the current draft, which reads: "Member States are called upon to prohibit any attempts to create human life through cloning processes and any research intended to achieve that aim." So we are once again back to a situation where states are having to make their own decisionlikely on a mixture of political, religious, and some scientific grounds-on when (individual?) "human life" begins.

What can be learnt from this experiment in creating a universal bioethical standard? First of all, if no meaningful universal agreement can be reached on reproductive cloning, at least not at the level of the United Nations, the prospect for reaching a global consensus on other issues in bioethics is rather bleak. It will be interesting to see if UNESCO will have more luck in its development of a declaration on universal norms on bioethics, which is currently in preparation. But no matter what the outcome is, it certainly makes us aware of the need to foster a genuine, world-wide discourse on bioethical issues (rather than leaving the field to political power games), which may be even more important than reaching immediate substantive conclusions. Developing rules and a language for such a "global discourse" is not trivial but may rather be one of the most important challenges for bioethics these days.

Another question that haunts the whole debate is: Why on earth devote so much time, energy and resources to the ethics of cloning rather than any other topic? Should the UN be bound up with this rather than other issues that seem as least as pressing? And isn't it peculiar that bioethics is associated in the minds of many people with cloning and embryos rather than questions of fair access to health care systems for these embryos once they have grown into adult human beings? Not only the item on the agenda, but the agenda itself is an issue that merits debate.

$J$ Med Ethics 2005;31:63. doi: 10.1136/jme.2004.011551

Correspondence to: Nikola Biller-Andorno, Institute of Medical Ethics, Charite - University Medicine, Berlin, Germany; billerandornon@ who.int 